\title{
Anxiety and depression in women undergoing infertility treatment
}

\author{
Paulina Gdańska ${ }^{1,2}$, Ewa Drozdowicz-Jastrzębska³ , Barbara Grzechocińska1, \\ Maria Radziwoń-Zaleska ${ }^{3}$, Piotr Węgrzynn ${ }^{2}$, Mirosław Wielgoś ${ }^{1}$ \\ ${ }^{1} 7^{\text {st }}$ Department of Obstetrics and Gynecology, Medical University of Warsaw, Warsaw, Poland \\ ${ }^{2}$ Department of Obstetrics and Perinatology, Medical University of Warsaw, Warsaw, Poland \\ ${ }^{3}$ Department of Psychiatry, Medical University of Warsaw, Warsaw, Poland
}

\begin{abstract}
Infertility is a significant problem for millions of couples. Recently more attention is being paid to the relationship between infertility treatment with the use of Assisted Reproductive Techniques and the presence of mental disturbances, of which anxiety and depression are the most common. We present a review of recent studies evaluating the influence of anxiety and depression on fertility treatment outcomes and the effect of Assisted Reproductive Techniques treatment on the presence of anxiety and depression among women. The studies show conflicting results concerning the effect of anxiety on Assisted Reproductive Techniques treatment outcomes, but most reveal that Assisted Reproductive Techniques treatment leads to an increased level of anxiety, especially in cases of treatment failure and longer durations of treatment. Most studies do not show a relationship between depression and Assisted Reproductive Techniques treatment outcomes, but it seems that severe depression can lead to lower rates of pregnancy during infertility treatment with Assisted Reproductive Techniques. Moreover, women who become pregnant after Assisted Reproductive Techniques treatment seem to have an increased risk of depression in later life.
\end{abstract}

Key words: infertility, assisted reproductive techniques, anxiety, depression

Ginekologia Polska 2017; 88, 2: 109-112

\section{INTRODUCTION}

Infertility is a substantial healthcare and social problem and according to the World Health Organization's (WHO) estimates from a 2010 study, it affects approximately 45 million couples worldwide [1]. Infertility is defined as the inability of a couple to conceive a child within 12 months of regular sexual activity without using any contraceptive methods [2]. Within the last few decades, the implementation of Assisted Reproductive Techniques (ART), such as in-vitro fertilization (IVF), intracytoplasmatic sperm injection (ICSI) or intrauterine insemination (IUI), brought advances in infertility treatment by increasing the number of pregnancies in infertile couples [3]. Each year the number of couples deciding to pursue ART treatment increases, yet the diagnosis of infertility and undergoing potentially long-term treatment giving uncertain results is often associated with a psychological burden [4]. As a result, patients undergoing infertil- ity treatment frequently experience symptoms of anxiety and depression of different intensity. The role of mental disturbances as possible causes for impaired fecundity has been discussed for many years [5], but the exact relationship between mental state and fertility and the influence of mental state on fertility treatment outcomes remain unclear. It seems noteworthy, that only slightly more than half of patients experiencing infertility seek medical advice [6] and the worsening of mental health can further reduce the chances of making the decision to pursue infertility treatment.

Patients with infertility often present symptoms of stress and adaptive disorders, of which anxiety and depression are the most common. Anxiety is a normal adaptive response of the organism to danger or stressful events [7]. Anxiety disorders belong to the most common mental disturbances and have a similar prevalence in different populations and cultures [8]. In the case of a high intensity of anxiety or its 
inadequacy to the causal trigger, it is considered as pathology and requires medical treatment.

Depressive disorders are common and constitute a significant healthcare problem. They are currently the fourth leading cause of disability worldwide and the WHO estimates, that in 2020 they will be ranked in the second place, behind cardiovascular disorders. The most common symptoms of depression include lowered mood, lowered activity, loss of interest and an inability to experience pleasure from joyful activities [9]. Clinical depression occurs in 5-10\% of the population, yet even as much as $30 \%$ of the population suffers from depressive symptoms of different intensity throughout their lives [7].

Most studies focusing on the relationship between infertility and mental state are carried out on small study groups and some large-population studies present conflicting results. It seems however that stress related to infertility can negatively affect the outcomes of infertility treatment. Infertile women seem to be more affected by stress compared to their male partners [10-14], which may be due to their direct involvement in invasive ART procedures. In our work we present a review of current literature on anxiety and depressive disorders in women undergoing infertility treatment, including the influence of anxiety and depression on infertility treatment outcome and the effect of ART procedures on the risk of anxiety and depression.

\section{EPIDEMIOLOGY}

There are substantial disparities in terms of the prevalence and incidence of anxiety and depressive disorders among patients undergoing infertility treatment in the conducted studies. In a study by Ramezanzadeh et al. $(n=370)$, $86.8 \%$ of women had anxiety and $40.8 \%$ of women suffered from depression. There was a relationship between anxiety and depression and the duration of infertility and the disorders were most prevalent at 4-6 years since the diagnosis of infertility [15]. A study of the Italian population of infertile couples $(n=100)$ revealed that $14.7 \%$ of women and $4.5 \%$ of men had anxiety [11], whereas the authors of a population-based study in Denmark found severe depression to be present in $11.6 \%$ of women and $4.3 \%$ of men undergoing infertility treatment [15].

\section{THE INFLUENCE OF ANXIETY ON THE EFFECTS OF INFERTILITY TREATMENT}

In the past decades multiple authors have suggested a model of "psychogenic infertility". According to this theory, factors such as personality traces, family relations, sexual disorders, feeling of guilt, an urge need to possess a baby or even the fear of labour would directly or indirectly lead to reduced fertility [4]. The new approach departs from the concept of "psychogenic infertility" and focuses on the complex relationship between fertility and psychological and social factors [5]. The current models take into account stress and its possible influence on fertility via the activation of hypothalamus-pituitary-adrenal (HPA) axis. In one of the studies, the relationship of HPA axis, the level of anxiety and the efficacy of IVF treatment were evaluated [17]. The study group consisted of 264 women undergoing IVF treatment. The authors found a statistically significant increase in the level of norepinephrine and cortisol on the oocyte retrieval day compared to an assessment before commencing the IVF procedure. There was also a relationship between the level of serum cortisol and the intensity of state anxiety in the psychometric questionnaires. Moreover, women who became pregnant initially had lower levels of norepinephrine and cortisol in the serum and intracellular fluid [17]. In a pilot study on a group of 44 women, an increased level of anxiety in the psychometric scales was found in women undergoing IVF treatment during the whole course of therapy [14]. It was also found that women initially who had a lower level of anxiety before the oocyte retrieval, had a higher pregnancy rate [14].

Conversely, some studies do not confirm the relationship between anxiety and the efficacy of infertility treatment $[18,19]$, including a meta-analysis of 31 prospective studies. In the study, researchers from Denmark found no association between anxiety disorders and the effects of ART treatment [20]. However, the authors underline a large heterogeneity of the study groups and a fairly low number of studies included in the meta-analysis.

\section{THE INFLUENCE OF INFERTILITY AND ART PROCEDURES ON ANXIETY}

Many authors concentrate on the effect of infertility on the mental state. The diagnosis of infertility is a significant source of stress and undergoing ART therapy can bring up fear of not succeeding in an often long-term treatment, thus increasing the stress level. Although the results of current studies are conflicting, many researchers found an increased level of anxiety in couples undergoing infertility treatment. In a study by Reis et al. they found that couples commencing ART treatment for the first time had a higher state anxiety score compared to couples who took part in multiple ART cycles [12]. In a different study, a higher level of anxiety was found in women who failed to achieve a pregnancy via IVF procedure [19]. A study by Kahyaoglu Sut et al. showed that undergoing infertility treatment was related to a lower quality of life and a higher level of anxiety in a study group of 89 women [21]. One of the studies carried on the German population found that both women and men had a higher level of anxiety compared to the general population and women had a higher anxiety level compared to men [13]. Moreover, the level of anxiety increased during the 
course of infertility treatment [13]. Some other studies also found a relationship between the duration of infertility and increased level of anxiety [11,22]. Conversely, large population-based studies carried on the Scandinavian population did not confirm a significant influence of ART treatment on the prevalence of anxiety disorders. A study on a group of 98320 Danish women showed no relationship between the results of infertility treatment and the risk of anxiety disorders [23]. One of the limitations of the study is that it only included those women, who were hospitalized due to their anxiety disorders, whereas most of anxiety disorders are treated in outpatient clinics. Another study conducted in Norway on a group of 9200 women evaluated self-reported health, functional impairment, the presence of anxiety and depression and the quality of life in a group of infertile women compared to women without infertility problems and among infertile women with or without a child. Childless infertile women reported more health-related complaints and a lower life satisfaction, yet there were no significant differences in the level of anxiety compared to women with a child, either with or without a history of infertility [24]. Authors of a different study on a group of 530 women in Sweden evaluated long-term effects of IVF procedure on the mental state. They found that the majority of women who underwent IVF did not have higher rates of mental illness after two decades of the treatment, but those women who remained childless and/or without a partner had a higher risk of phobic anxiety [25].

\section{THE INFLUENCE OF DEPRESSION ON THE EFFECTS OF INFERTILITY TREATMENT}

The exact causal relationship between mental state and fertility remains unclear, yet the results of some studies suggest that depression could lead to decreased fertility and lower rates of pregnancy in patients undergoing an IVF procedure. A large cohort study on the Danish population revealed that women with depression undergoing ART treatment had a lower rate of commenced ART cycles compared to women without diagnosed depression [26]. Moreover, women with a prior diagnosis of depression had a lower mean number of live births after ART procedures compared to women without depression. The study limitations include the fact that only those cases of depression that required hospitalization were taken into account. Other studies however did not find a relationship between depression and lower chances of successful infertility treatment. In a study conducted in the USA on a group of 202 women undergoing an IVF procedure for the first time, a diagnosis of depression had no effect on the efficacy of IVF [19]. Also a meta-analysis by Mathiessen et al. did not reveal a significant influence of depression diagnosed before ART therapy on the number on achieved pregnancies [20].

\section{THE INFLUENCE OF INFERTILITY AND ART ON DEPRESSION}

There is increasing interest in the potential influence of infertility treatment on the risk of depressive disorders. A study conducted in Sweden $(n=520)$ showed that women who underwent IVF therapy in the past had a higher risk of depression after 20-23 years, regardless of whether they gave birth in the meantime or remained childless [25]. A different study by Roastad et al. $(n=9200)$ on the Norwegian population did not reveal significant differences in the incidence of depression among infertile women compared to those without infertility problems and in infertile women who were childless compared to those with children [24]. Another study on depression among couples undergoing ART treatment $(n=98)$ showed that couples undergoing subsequent ART cycles presented a higher level of depressive symptoms compared to couples beginning ART therapy for the first time. Furthermore, women had a higher intensity of depressive symptoms compared to men [12]. Interesting results came from a study conducted by Sejbaek et al. in Denmark on a group of 41050 women who were treated with ART procedures. Depression was diagnosed in a total of 552 women. The study revealed that women who remained childless had a lower risk of depression compared to those who gave birth after ART procedures. The risk of depression among patients who became pregnant was highest during the first 42 days postpartum and, although lower, remained elevated within the first year after parturition as well as after one year since labour [27].

\section{SUMMARY}

Infertility remains a significant social problem and a challenge for healthcare systems worldwide. As the number of infertile couples deciding to undergo ART treatment is increasing, more attention is being paid to the negative consequences of stress related to infertility and invasive reproductive procedures.

Although it remains unclear whether an increased level of anxiety influences the effectiveness of ART procedures, it undoubtedly leads to a lower quality of life amongst infertile couples. It also seems that undergoing ART treatment leads to an increased anxiety level, especially in cases of treatment failure and longer duration of the therapy. Furthermore, most studies reveal higher levels of anxiety and depression among women compared to men during infertility treatment.

There are some uncertainties concerning the influence of depression on the results if infertility treatment, yet it seems that severe depression occurring prior to commencing ART treatment can be related to lower rates of pregnancies. Despite some discrepancy in the results of the studies, the evidence suggests that achieving pregnancy with the 
use of ART is related with higher risk of depression in the early postpartum period as well as in the future.

Further studies are needed to evaluate the relationship between mental state and fertility, yet it seems to be a problem that deserves particular attention. Psychological support and early psychiatric treatment in case of severe mental issues could lead to an improvement in the health and the quality of life of infertile couples and could potentially improve infertility treatment outcomes.

\section{REFERENCES}

1. Mascarenhas MN, Flaxman SR, Boerma T, [et al.]. National, Regional, and Global Trends in Infertility Prevalence Since 1990: A Systematic Analysis of 277 Health Surveys. PLoS Med. 2012;9(12):1-12. doi:10.1371/journal. pmed.1001356.

2. World Health Organization (WHO). International Regulatory Co-operation. 2016: 198-199, doi: 10.1787/9789264244047-59-en.

3. Dunn AL, Stafinski T, Menon D. An international survey of assisted reproductive technologies (ARTs) policies and the effects of these policies on costs, utilization, and health outcomes. Health Policy. 2014; 116(2-3): 238-263, doi: 10.1016/j.healthpol.2014.03.006, indexed in Pubmed: 24698476.

4. Podolska MZ, Bidzan M. Infertility as a psychological problem. Ginekol Pol. 2011; 82(1): 44-49, indexed in Pubmed: 21469521.

5. Holka-Pokorska J, Jarema M, Wichniak A. Clinical determinants of mental disorders occurring during the infertility treatment. Psychiatr Pol. 2015; 49(5): 965-982, doi: 10.12740/PP/35958, indexed in Pubmed: 26688847.

6. Boivin J, Schmidt L. Infertility-related stress in men and women predicts treatment outcome 1 year later. Fertil Steril. 2005; 83(6): 1745-1752, doi: 10.1016/j.fertnstert.2004.12.039, indexed in Pubmed: 15950646.

7. Oxford Handbook of Psychiatry. Oxford Medicine Online. 2013, doi: 10.1093/med/9780199693887.001.0001.

8. Rybakowski J, Pużyński S, Wciórka J. Psychiatria. Tom 2 - Psychiatria kliniczna. 2nd ed. Elsevier Uban \& Partner, 2011

9. World Health Organization: The ICD-10 classification of mental and behavioural disorders: clinical descriptions and diagnostic guidelines. Geneva: World Health Organization, 1992

10. Baghianimoghadam $\mathrm{MH}$, Aminian $\mathrm{AH}$, Baghianimoghadam $\mathrm{B}$, et al. Mental health status of infertile couples based on treatment outcome. Iran J Reprod Med. 2013; 11(6): 503-510, indexed in Pubmed: 24639785.

11. Chiaffarino F, Baldini MP, Scarduelli $C$, et al. Prevalence and incidence of depressive and anxious symptoms in couples undergoing assisted reproductive treatment in an Italian infertility department. Eur J Obstet Gynecol Reprod Biol. 2011; 158(2): 235-241, doi: 10.1016/j. ejogrb.2011.04.032, indexed in Pubmed: 21641108.

12. Reis $S$, Xavier MR, Coelho $R$, et al. Psychological impact of single and multiple courses of assisted reproductive treatments in couples: a comparative study. Eur J Obstet Gynecol Reprod Biol. 2013; 171(1): 61-66, doi: 10.1016/j.ejogrb.2013.07.034, indexed in Pubmed: 23928476.

13. Schaller MA, Griesinger G, Banz-Jansen C. Women show a higher level of anxiety during IVF treatment than men and hold different concerns: a cohort study. Arch Gynecol Obstet. 2016; 293(5): 1137-1145, doi: 10.1007/s00404-016-4033-x, indexed in Pubmed: 26884350.

14. Turner K, Reynolds-May MF, Zitek EM, et al. Stress and anxiety scores in first and repeat IVF cycles: a pilot study. PLoS ONE. 2013; 8(5): e63743, doi: 10.1371/journal.pone.0063743, indexed in Pubmed: 23717472.

15. Ramezanzadeh F, Aghssa MM, Abedinia N, et al. A survey of relationship between anxiety, depression and duration of infertility. BMCWomens Health. 2004; 4(1): 9, doi: 10.1186/1472-6874-4-9, indexed in Pubmed: 15530170

16. Peterson BD, Sejbaek CS, Pirritano M, et al. Are severe depressive symptoms associated with infertility-related distress in individuals and their partners? Hum Reprod. 2014; 29(1): 76-82, doi: 10.1093/humrep/det412, indexed in Pubmed: 24256990.

17. An $\mathrm{Y}$, Wang $\mathrm{Z}$, Ji $\mathrm{H}$, et al. Pituitary-adrenal and sympathetic nervous system responses to psychiatric disorders in women undergoing in vitro fertilization treatment. Fertil Steril. 2011; 96(2): 404-408, doi: 10.1016/j. fertnstert.2011.05.092, indexed in Pubmed: 21722893.

18. Hashemi S, Simbar M, Ramezani-Tehrani F, et al. Anxiety and success of in vitro fertilization. Eur J Obstet Gynecol Reprod Biol. 2012; 164(1): 60-64, doi: 10.1016/j.ejogrb.2012.05.032, indexed in Pubmed: 22727918.

19. Pasch LA, Francisco S, Gregorich SE, et al. HHS Public Access. 2016; 98(2): 459-464, doi: 10.1016/j.fertnstert.2012.05.023.Psychological.

20. Matthiesen SMS, Frederiksen Y, Ingerslev HJ, et al. Stress, distress and outcome of assisted reproductive technology (ART): a meta-analysis. Hum. Reprod. 2011;26(10): 2763-2776, doi: 10.1093/humrep/der246, indexed in Pubmed: 21807816.

21. Kahyaoglu Sut H, Balkanli Kaplan P. Quality of life in women with infertility via the FertiQoL and the Hospital Anxiety and Depression Scales. Nurs Heal Sci. 2015;17(1):84-89. doi:10.1111/nhs.12167.

22. Hashemieh C, Neisani Samani L, Taghinejad H. Assessment of Anxiety in Pregnancy Following Assisted Reproductive Technology (ART) and Associated Infertility Factors in Women Commencing Treatment. Iran Red Crescent Med J. 2013; 15(12): e14465, doi: 10.5812/ircmj.14465, indexed in Pubmed: 24693397.

23. Baldur-Felskov B, Kjaer SK, Albieri V, et al. Psychiatric disorders in women with fertility problems: results from a large Danish register-based cohort study. Hum Reprod. 2013; 28(3): 683-690, doi: 10.1093/humrep/des422, indexed in Pubmed: 23223399.

24. Rostad B, Schmidt L, Sundby J, et al. Infertility experience and health differentials - a population-based comparative study on infertile and non-infertile women (the HUNT Study). Acta Obstet Gynecol Scand. 2014; 93(8): 757-764, doi: 10.1111/aogs.12404, indexed in Pubmed: 24773205.

25. Vikström J, Josefsson A, Bladh $\mathrm{M}$, et al. Mental health in women 2023 years after IVF treatment: a Swedish cross-sectional study. BMJ Open. 2015; 5(10): e009426, doi: 10.1136/bmjopen-2015-009426, indexed in Pubmed: 26510732.

26. Sejbaek CS, Hageman I, Pinborg A, et al. Incidence of depression and influence of depression on the number of treatment cycles and births in a national cohort of 42,880 women treated with ART. Hum. Reprod. 2013; 28(4): 1100-1109, doi: 10.1093/humrep/des442, indexed in Pubmed: 23300199.

27. Sejbaek CS, Pinborg A, Hageman I, et al. Are repeated assisted reproductive technology treatments and an unsuccessful outcome risk factors for unipolar depression in infertile women? Acta Obstet Gynecol Scand. 2015; 94(10): 1048-1055, doi: 10.1111/aogs.12705, indexed in Pubmed: 26234480 